\title{
Measurement and simulation of the new liquid organic scintillator response to fast neutrons
}

\author{
Jaroslav Jánský ${ }^{1}$, Jiř́ Janda ${ }^{2}$, Zdeněk Matěj ${ }^{3}$, Filip Mravec ${ }^{3}$, Michal Koštál ${ }^{4}$, \\ František Cvachovec ${ }^{1}$ \\ ${ }^{1}$ University of Defence, Faculty of Military Technology, Department of Mathematics and Physics, \\ Brno, Czech Republic \\ ${ }^{2}$ University of Defence, NBC Defence Institute, Vyškov, Czech Republic \\ ${ }^{3}$ Masaryk University, Faculty of Informatics, Brno, Czech Republic \\ ${ }^{4}$ Research Centre Řež, Husinec-Ǩež, Czech Republic \\ Corresponding author: jaroslav.jansky@unob.cz
}

\begin{abstract}
Liquid organic scintillators are important devices for measurements of neutron radiation. This work aims to develop and optimize the composition of liquid organic scintillators so it can be used for fast neutron spectrometry. As the neutron radiation is usually accompanied with $\gamma$ ray radiation, it is important to have quality $\gamma / \mathbf{n}$ discrimination. The new cocktail for house made liquid organic scintillator is prepared and studied with intention of being able to separate gamma and neutron for neutron energies above $0.5 \mathrm{MeV}$ while keeping lower constraints on practical use (e.g., sealing because of oxygen) than commercial liquid scintillators. In preceding work the composition of liquid scintillators was optimized. Two twocomponent scintillators were selected for further studies. Solvent DIPN (Di-iso-propyl-naphthalene Mixed Isomers) is selected for both. First is mixed with luminophore PYR (1Phenyl-3-(2,4,6-trimethyl-phenyl)-2-pyrazoline) of concentration $5 \mathrm{~g} / \mathrm{l}$. Second is mixed with luminophore THIO (2,5-Bis(5-tert-butyl-benzoxazol-2-yl)thiophene) of concentration $5 \mathrm{~g} / \mathrm{l}$. In this work the response of scintillator to monoenergetic beam of neutrons was measured for multiple neutron energies at PTB in Braunschweig. The two parameter spectrometric system NGA-01 is used to analyze the energy and discrimination characteristics. 137 Cs and 60 Co are used as radiation sources for calibration with pure $\gamma$ rays. Then the response of scintillator for same neutron energies was simulated using GEANT4. The dissipated energy in the scintillator in response to monoenergetic neutrons is obtained. Both, measured and simulated responses are compared. Functional dependence for yield of recoiled products is estimated. It is seen that main recoil product hydrogen proton is well observed in both. From the edge of proton response one can assume the yield for given neutron energy. The recoiled carbon ion (from elastic collision) is on the other side difficult to observe in measured results but clearly seen in dissipated energy plots. It suggests that yield of carbon ion is very small relatively to proton yield. These results will serve as basis for response function evaluation of scintillator which is necessary for evaluation of unknown neutron spectra from measurements with scintillator.
\end{abstract}

\section{INTRODUCTION}

Liquid organic scintillators are important devices for measurements of neutron radiation. Organic liquid scintillators can consist simply of an organic solvent and a luminophore. A third constituent is sometimes added as a wavelength shifter to fit the emission spectrum to better match the spectral response of common photomultiplier tubes. In certain applications, large-volume detectors with dimensions of several meters may be required. In these cases, the liquid scintillator is often the only practical choice because of the cost. Liquid scintillators are expected to be more resistant to radiation damage effects than crystalline or plastic scintillators, because of their lack of a solid structure that could be damaged by an exposure to intense radiation.

This work aims to develop and optimize the composition of liquid organic scintillators so it can be used for fast neutron spectrometry. As the neutron radiation is usually accompanied with $Y$ ray radiation, it is important to have quality $\gamma / n$ discrimination. The responses of scintillator to monoenergetic neutrons are shown. These results are planned to be used in future for development of response matrix for our scintillators.

\section{II.EXPERIMENTAL DESCRIPTION}

The two scintillators are used in this work. Two twocomponent scintillators were selected for these studies. Solvent DIPN (Di-iso-propyl-naphthalene Mixed Isomers) is selected for both. First is mixed with luminophore PYR (1Phenyl- 3 - ( 2 , 4 ,6 - trimethyl - phenyl ) - 2 - pyrazoline) of concentration $5 \mathrm{~g} / \mathrm{l}$. Second is mixed with luminophore THIO ( 2 , 5 - Bis ( 5 - tert - butyl - benzoxazol - 2 - yl ) thiophene) of concentration $5 \mathrm{~g} / \mathrm{l}$.

In this work the response of scintillator to monoenergetic beam of neutrons was measured for multiple neutron energies at PTB in Braunschweig. The two parameter spectrometric system NGA-01 is used to analyze the energy and discrimination characteristics. $137 \mathrm{Cs}$ and 60 Co are used as radiation sources for calibration with pure $\gamma$ rays.

For numerical simulations GEANT 4 version 10.0.6 was 
used. Physics list Shielding was used as it supports the measured cross section data for neutron collisions below 20 $\mathrm{MeV}$. The simulated geometry consisted of $10 \mathrm{~cm}$ cube box with vacuum. The scintillator cylinder with radius $1 \mathrm{~cm}$ and height $4.5 \mathrm{~cm}$ is placed inside. The particle source is placed on the axis of symmetry of cylinder $1 \mathrm{~cm}$ ahead.

The scinitllator density is considered to be $0.949 \mathrm{~g} / \mathrm{cm}^{3}$. Atom ratio for plain DIPN $\mathrm{H}: \mathrm{C}$ is 1.25. Including the luminophore PYR shifts the $\mathrm{H}: \mathrm{C}$ ratio to 1.2494 and nitrogen is present in ratio $\mathrm{N}: \mathrm{C} 0.0005$. The results of GEANT4 simulations are presented in form of absorbed (deposited) energy inside the scintillator after each of the source neutrons leaves the simulation domain.

\section{RESULTS}

In this section we first present the measured responses of our two scintillators to the monoenergetic beams.

First the response of the scintillator with THIO luminophore to neutrons of energy $1.5 \mathrm{MeV}$ is shown in Figure 1 . Horizontal axis denotes the energy in electron equivalent units. Vertical axis denotes the pulse height spectra.

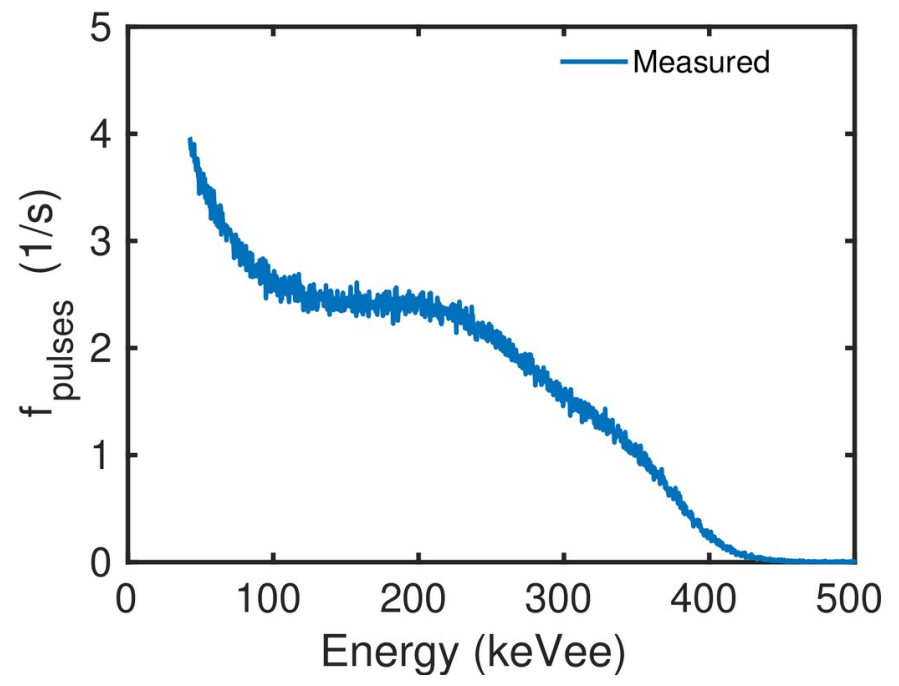

Fig 1: Response of scintillator with THIO luminophore to neutrons of energy $1.5 \mathrm{MeV}$.

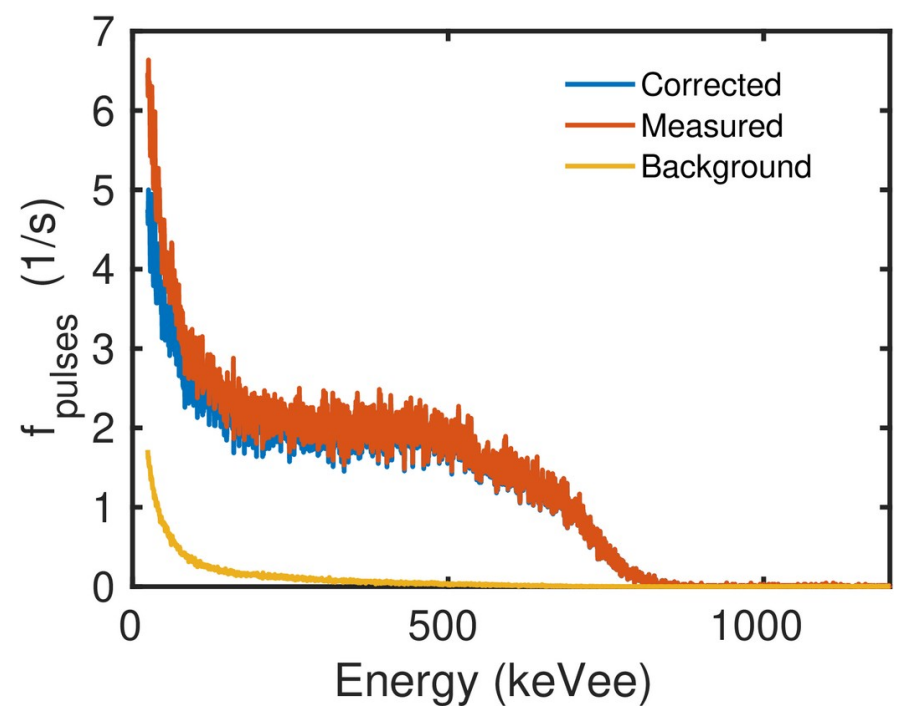

Fig 2: Response of scintillator with THIO luminophore to neutrons of energy $2.5 \mathrm{MeV}$.

Then the response of the scintillator with THIO luminophore to neutrons of energy $2.5 \mathrm{MeV}$ is shown in Figure 2. The measured pulse height spectra is shown with red line. Then the measurement with shadow cone to subtract the scattered background neutrons is shown with yellow line. And finally the corrected pulse height spectrum is shown with blue line.

Then the response of the scintillator with THIO luminophore to neutrons of energy $19 \mathrm{MeV}$ is shown in Figure 3.

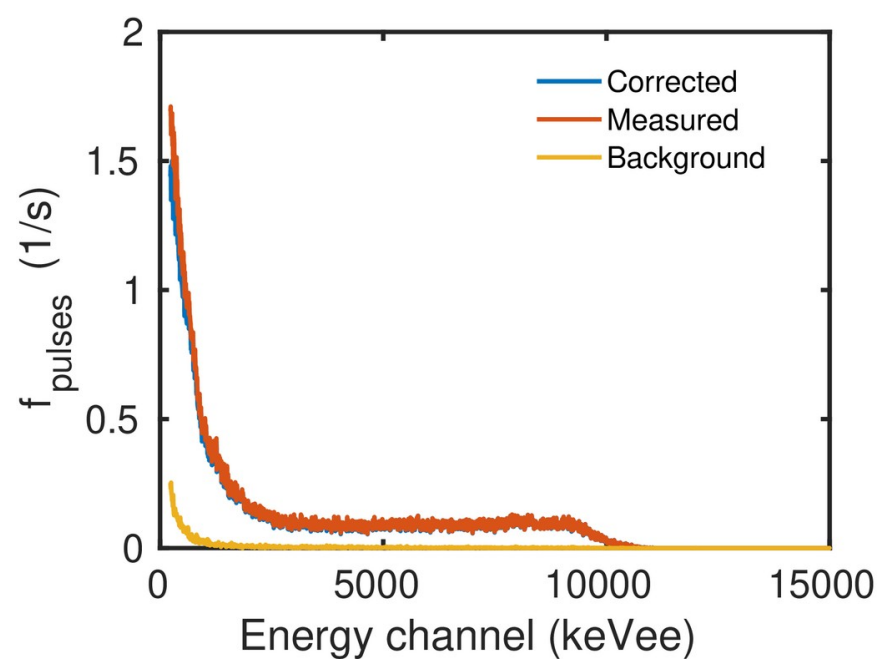

Fig 3: Response of scintillator with THIO luminophore to neutrons of energy $19 \mathrm{MeV}$.

The response to scintillator with PYR luminophore is measured twice for energy $2.5 \mathrm{MeV}$ and once for energy 19 $\mathrm{MeV}$. The pulse height spectra are shown below in Figure 4,5 and 6.

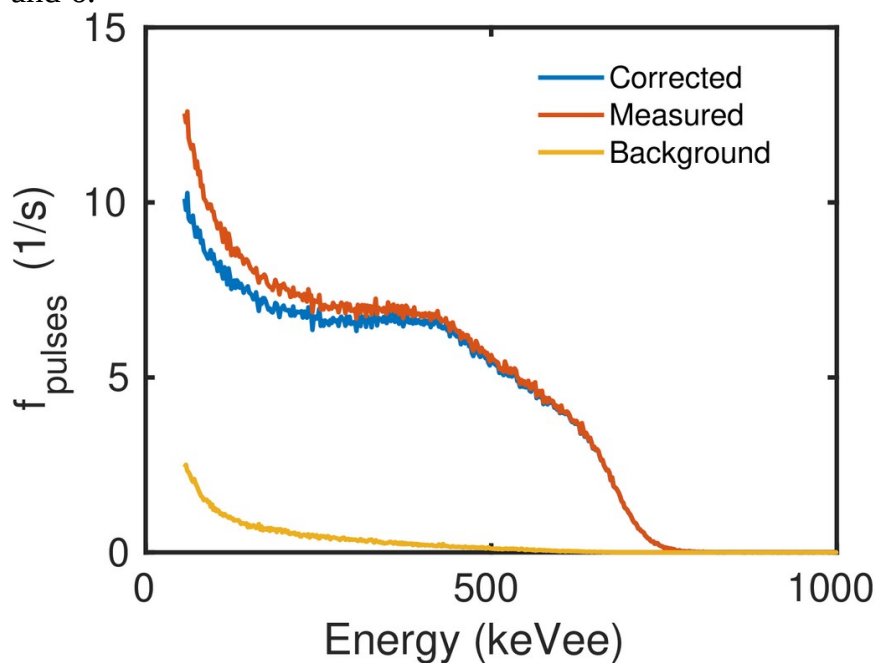

Fig 4: Response of scintillator with PYR luminophore to neutrons of energy 2.5 MeV. 


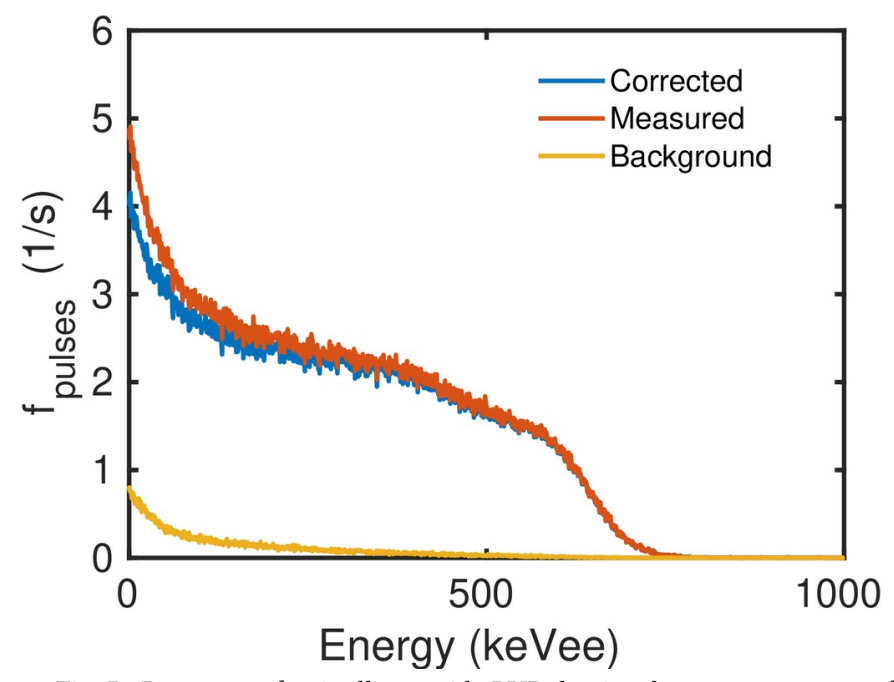

Fig 5: Response of scintillator with PYR luminophore to neutrons of energy $2.5 \mathrm{MeV}$.

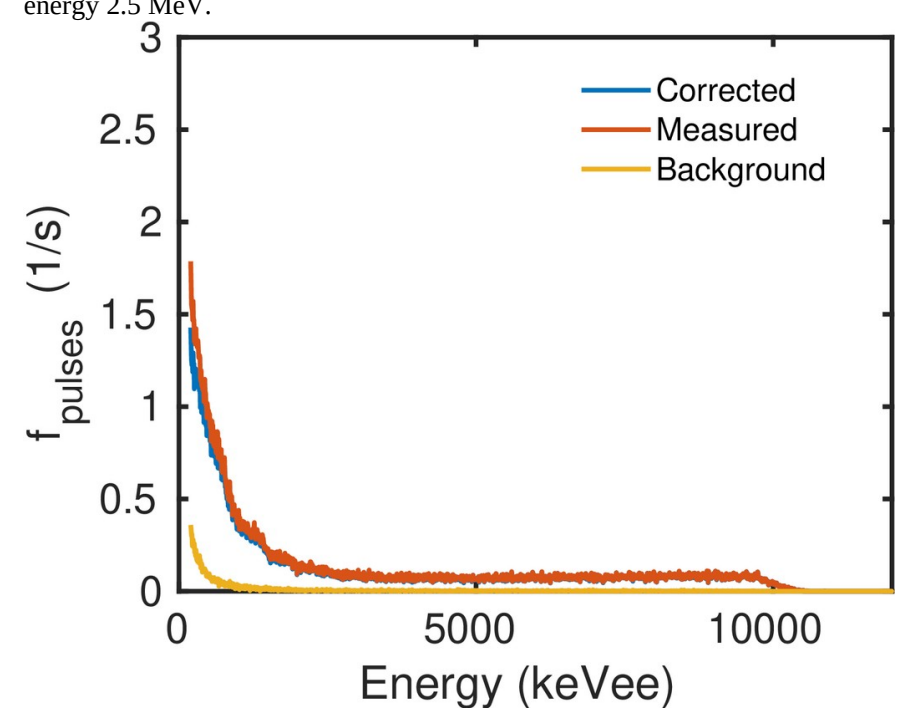

Fig 6: Response of scintillator with PYR luminophore to neutrons of energy $19 \mathrm{MeV}$.

The simulated deposited energy for neutrons of energy 1.5 and $2.5 \mathrm{MeV}$ using GEANT4 are shown in Figures 7 and 8.

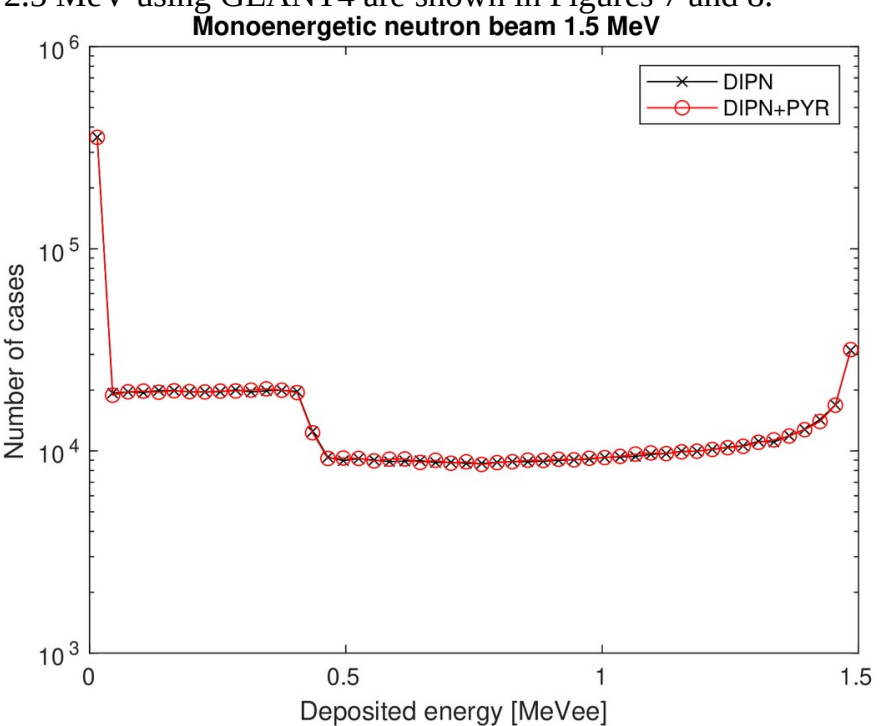

Fig 7: Deposited energy of scintillator with only DIPN and DIPN with luminophore to neutrons of energy $2.5 \mathrm{MeV}$.

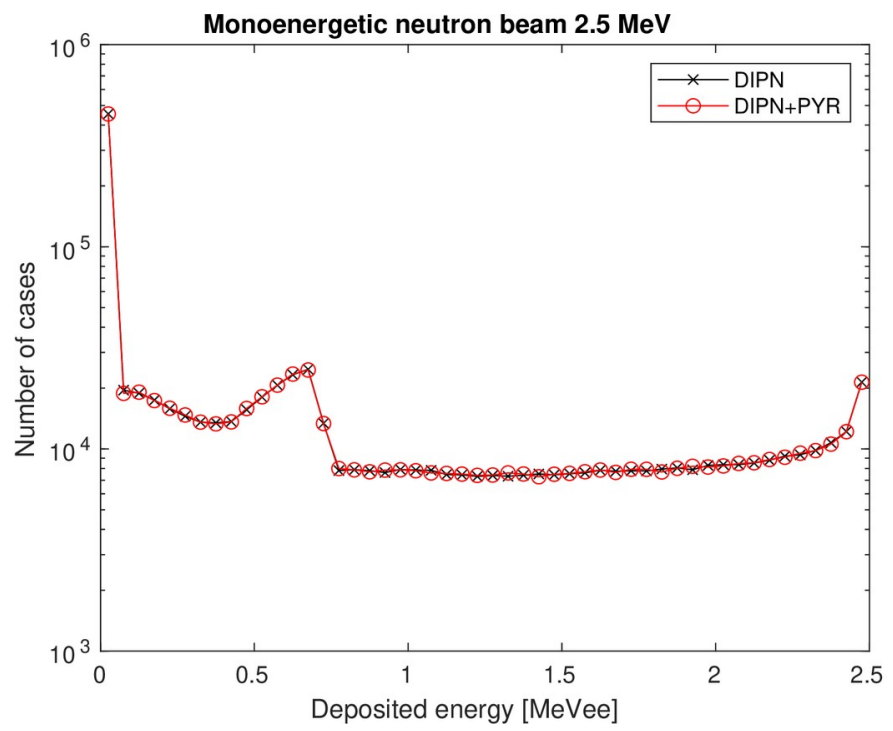

Fig 8: Deposited energy of scintillator with only DIPN and DIPN with luminophore to neutrons of energy $2.5 \mathrm{MeV}$.

It clear to observe deposited energy due to carbon ions from increased spectra below $0.4 \mathrm{MeV}$ and $0.6 \mathrm{MeV}$ for $1.5 \mathrm{MeV}$ neutron source, respectively $2.5 \mathrm{MeV}$. However this pattern is not observed in experimental results suggesting that light yield of recoiled carbon ion is much smaller than light yield of recoiled proton after neutron elastic collisions.

The summary of light yield function from experimental measurements is shown in Figure 9. The line represents theoretical function for gamma energy which is identical to electron equivalent energy. The points represents results from above measured responses and they all show that neutrons produce several times less light than gamma rays of same energy.

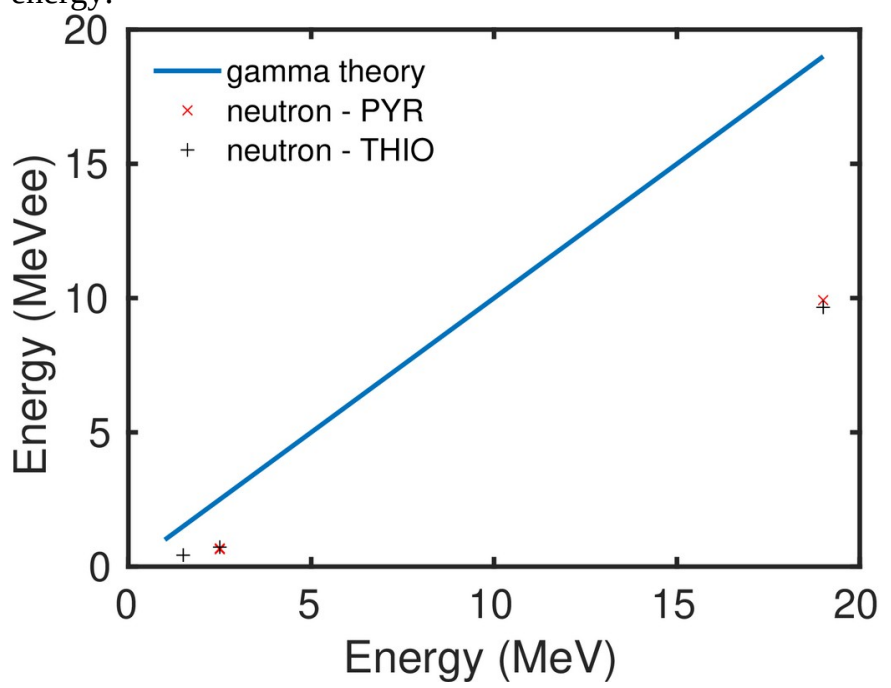

Fig 9: Summary of the light yield function relating the neutron energy to electron equivalent energy.

\section{CONCLUSIONS}

In this work the response of scintillator to mono-energetic beam of neutrons was measured for multiple neutron energies and compared to the dissipated energy response of scintillator for same neutron energies simulated using GEANT4. Functional dependence for yield of recoiled products is 
estimated. It is seen that main recoil product hydrogen proton is well observed in both. From the edge of proton response one can assume the yield for given neutron energy. The recoiled carbon ion (from elastic collision) is on the other side difficult to observe in measured results but clearly seen in dissipated energy plots.

\section{ACKNOWLEDGEMENT}

This research work has been carried out within the ADAR project (Accelerator Driven Advanced Reactor). The authors gratefully acknowledge the financial support from the Ministry of Education, Youth, and Sports of the Czech Republic under the INTER-ACTION research program (project No. LTAUSA18198). The authors gratefully acknowledge the financial support from the grant VAROPS (DZRO FVT 3) granted by the Ministry of Defence of the Czech Republic. This work has received funding from the Euratom research and training programme 2014-2018 under grant agreement No 847594 (ARIEL). 